\title{
Windows on the Human Brain and the Neurobiology of Psychiatric Illness
}

Significant advances have been made in understanding the molecular and cellular underpinnings of neuronal transmission, plasticity and function of the brain, based largely on studies in animal models. Discoveries made in experimental animals provide novel insights into the mechanisms underlying the function of the human brain, and are often utilized to form hypotheses about neurochemical imbalances that lead to psychiatric illnesses. However, unlike many other fields of modern medicine where hypotheses can be tested and data can be collected from the diseased tissue via biopsy in the living patient, research on the biology of disorders of the human brain are not afforded this luxury. Studies of live patients are limited to analysis of metabolites in the blood, which is readily available but does not provide information that directly reflects the brain, or cerebral spinal fluid, which provides a more direct measure of brain neurochemistry but is more difficult to obtain and is still only a reflection of spillover of metabolites from millions of different cell types. Studies to determine the genetic basis of major psychiatric disorders are also being conducted, but because of the complexity of these illnesses and the contribution of environmental factors this approach is progressing at a slow rate.

The inability to sample and analyze the diseased tissue in human brain has severely limited elucidation of the neurobiology of mental illnesses and this explains why the field of Psychiatry has turned to analysis of postmortem tissue. Based on the increased number of investigators requesting specimens and a recent announcement of supplemental funding for NIMH grants there has been a renewed interest in postmortem studies over the past decade. Although postmortem studies were criticized early on for confounds and problems too numerous to control for, there has been continued interest in pursuing this avenue because it provides one of the few approaches that we have to identify the synaptic and cellular events, as well as molecular determinants, un- derlying psychiatric illnesses. Significant progress has been made in designing approaches for dealing with confounds facing postmortem research, and this is the subject of the perspective by David Lewis in this issue of Neuropsychopharmacology. Lewis addresses the problems and pitfalls encountered when using postmortem tissues, and highlights the potential payoff of well-designed studies.

Lewis focuses on 3 major areas that are critical for postmortem studies: 1) Well characterized brain specimens; 2) Well conceptualized or designed studies; and 3) Well controlled confounds. In each case, Lewis describes potential problems, often illustrated with specific examples, and then provides suggestions for improving or addressing each problem. The characterization of brain specimens receives the most attention as would be expected because the data obtained in postmortem studies are only as good as the collection and processing of the starting material. Lewis highlights the need for good clinical diagnosis and argues that the same high quality criteria used for clinical studies of patients should also be applied to postmortem investigations. This is clearly one of the most difficult tasks but suggestions for improvements, such as creating standardized, as well as new, diagnostics procedures are provided. In addition, neuropathological assessment, antemortem and postmortem factors, and toxicology are also discussed. A very nice section on postmortem factors, including several examples, highlights the need to look at each different molecular endpoint on an individual basis. Much of the work to characterize the influence of different factors on the stability (e.g. postmortem interval, tissue $\mathrm{pH}$ ) can be piloted in animal models. In addition, the possibility of creating a standardized brain bank of nonhuman primate tissue samples with different postmortem intervals is raised.

The concept or design of a study is another critical factor that is discussed in the Perspective. Lewis points 
out that it may not be impossible to answer certain questions with the tissue specimens available. In many cases this leads to a choice between not conducting a study or of obtaining data that are flawed. Because of the wide range of variables and limited number of postmortem samples available, it is often impossible to match tissues in a meaningful way. This highlights a major need in the field for collection of additional tissue specimens from both healthy and ill patients that will provide a greater resource base for postmortem work. Lewis also points out the critical need for the use of systematic, uniform random sampling (stereological principles) for postmortem analysis. This type of unbiased stereology is the accepted standard for cell counting in preclinical studies and should be the standard for human postmortem studies as well.

The last section addresses the control for confounds, including the influence of length of illness, comorbidity with other illnesses, and exposure to therapeutic drugs. The best way to control for these confounds is to have the appropriate controls, including samples from individuals who are ill for different lengths of time, who have one or more illnesses, or who are on or off medication at the time of death. Those controls are clearcut and straightforward, but require a large bank of specimens from healthy subjects and ill patients with a variety of backgrounds and environmental exposures. This is a clear limitation and again emphasizes the need for continued collection and development of postmortem brain banks. Another approach for dealing with some of these problems is to model confounds in rodents or nonhuman primates, for example, the influence of different types of medication.
Continued use and improvement of postmortem studies will contribute to the difficult task of elucidating the neurobiological abnormalities underlying major psychiatric illnesses such as anxiety, depression, and schizophrenia. Moreover, postmortem studies can be combined with genetic and brain imaging approaches in a complimentary fashion. For example, studies of brain tissue can determine the outcome of a genetic mutation that has been identified (i.e., is the expression of the mutated gene altered), or conversely can determine if altered expression of a novel gene identified by postmortem analysis results from a genetic abnormality (i.e., is the altered expression a result of a gene mutation). The latter possibility is especially exciting with the advent of DNA microarray approaches that allow for the analysis of thousands of genes on a single chip and is not limited to preconceived hypotheses. The ability to characterize molecular and cellular alterations will be accomplished at a more rapid rate when the number and quality of brain specimens is substantially increased and with continued improvements in the design of studies. Although this is an extremely complex and difficult task, the use of postmortem tissue as a component of multidisciplinary approaches offers the hope of improving the diagnosis and treatment debilitating psychiatric illnesses.

Ronald S. Duman, Ph.D. Division of Molecular Psychiatry Abraham Ribicoff Research Facilities Connecticut Mental Health Center Yale University School of Medicine New Haven, CT 